\title{
Análisis de métodos para evaluar el refuerzo a esfuerzo cortante con CFRP en vigas de hormigón armado
}

\author{
Method of analysis to evaluate the CFRP Shear- \\ Strengthened in Reinforced Concrete
}

\author{
C. Parra $^{(*)}$, E. Martínez-Conesa ${ }^{(*)}$, M. Valcuende ${ }^{(* *)}$, A. Garrido ${ }^{(*)}$
}

RESUMEN

En este trabajo de investigación se comparan diferentes modelos de cálculo de refuerzo a esfuerzo cortante mediante polímeros reforzados con fibras de carbono (CFRP) clasificados según el modo de fallo. En el diseño del refuerzo se tiene en cuenta la modificación del comportamiento de la viga por el refuerzo. Los estudios muestran que siempre que sea posible, la dirección principal de las fibras debe ser perpendicular a las fisuras de cortante. La contribución del refuerzo a la resistencia a cortante de la viga depende de los estribos de acero existentes en la viga original. Cuando aumenta el espesor del refuerzo la resistencia a cortante de la viga se incrementa. Esta relación tiende a ser lineal cuando la viga no está fisurada. Por último indicar que tanto el ángulo de inclinación de las fisuras, como el cortante resistido por el refuerzo, dependen del ángulo de inclinación de las fibras.

$605-2$

Palabras clave: Rehabilitación; refuerzo con fibras; esfuerzo a cortante; hormigón; fibra de carbono.

\section{SUMMARY}

Different models of shear-strengthened calculation by means of Carbon FiberReinforced Polymer (CFRP) are compared in this investigation works classified according to the way of failure. We take into account the modification of the behavior of the beam for the reinforcement in its design. The surveys show that as long as it is possible the main direction of the fibers must be perpendicular to the shear crakc. The contribution of the reinforcement to the resistance to shear-strengthened of the beam depends on the steel stirrups in the original beam. When the reinforcement strips thickness increases the resistance to shear-strengthened of the beam also increases. This relation tends to be lineal when the beam is not cracks. At last we must state that both the inclination angle of the crack and the shear-strengthened resisted by the reinforcement depend on the inclination angle of the fiber.
Keywords: restoration; strengthened with fiber; shear-strengthened; concrete; carbon fiber.

(*) Universidad Politécnica de Cartagena, Cartagena (España)

$(* *)$ Universitat Politècnica de València, Valencia (España)

Persona de contacto/Corresponding author: carlos.parra@upct.es (C. Parra) 


\section{INTRODUCCIÓN}

Actualmente, según el Ministerio de la Vivienda existen en España más 25 millones de viviendas. De ellas, la mitad supera los 30 años de antigüedad y cerca de 6 millones superan los 50 años. Este gran parque inmobiliario necesita diversas actuaciones de intervención, bien sea para asegurar la seguridad o para permitir adaptar su uso a nuevas necesidades. El refuerzo de estructuras de hormigón armado mediante materiales compuestos, en especial mediante polímeros reforzados con fibras (FRP), tiende a sustituir a sistemas de intervención más tradicionales, como los refuerzos mediante encolado de bandas de acero $(1,2)$. De hecho es posible encontrar una gran variedad de productos comerciales basados en materiales compuestos FRP en la industria de la construcción: los tendones de ARAPREE ( $)$ de SIREG, las varillas tipo POLYSTAL $(\mathbb{B}$ de POLYSTAL GmbH (Alemania), los laminados unidireccionales de fibra de carbono tipo CARBODUR ${ }^{\circledR}$ y WRAP ${ }^{\circledR}$ de SIKA (Suiza), las varillas tipo SPIFLEX $\mathbb{R}^{\mathrm{TM}}$, los tendones JONC J.T. ${ }^{\circledR}$ y el tejido TFC ${ }^{\circledR}$ (Francia).

En este trabajo se realiza un análisis de diferentes modelos de cálculo de refuerzo con FRP a cortante, cuyos resultados no son siempre convergentes. Así, Triantafillou (37), Deniaud y Cheng (8). Khalifa y Nanni (9-11) estiman que la efectividad del refuerzo a cortante depende principalmente del mecanismo de fallo, y basan su modelo analítico en un factor de eficiencia. Malek y Saadatmanesh $(12,13)$ plantean una formulación basada en la compatibilidad de deformaciones entre el tejido de refuerzo y la viga de hormigón en estado fisurado, proponiendo un método iterativo para conocer el ángulo de inclinación de las fisuras.

\subsection{Antecedentes}

En las últimas décadas ha surgido un interés especial en la búsqueda de materiales con características apropiadas para los proyectos arquitectónicos, donde es necesaria la aplicación de materiales resistentes y duraderos.

A partir de la década de los noventa, la investigación experimental en relación al

Tabla 1. Clasificación del refuerzo según el Japan Concrete Institute

\begin{tabular}{|c|c|c|}
\hline Técnica de reparación & Objetivo del Refuerzo & Ápliaciones \\
\hline \multirow{7}{*}{ Refuerzo por adherencia } & Flexión & $\begin{array}{c}\text { Pilares (puentes, edificios), } \\
\text { Vigas (puentes), vigas, } \\
\text { Forjados, chimeneas }\end{array}$ \\
\cline { 2 - 3 } & Cortante & $\begin{array}{c}\text { Pilares (puentes, edificios), } \\
\text { Vigas (puentes), vigas, } \\
\text { Forjados, chimeneas }\end{array}$ \\
\cline { 2 - 3 } & Compresión & Pilares (puentes, edificios) \\
\cline { 2 - 3 } & Prevención del deterioro & Chimeneas, túneles, postes \\
\hline
\end{tabular}

comportamiento estructural de los FRP como refuerzo en estructuras de hormigón, ha tenido un avance significativo (14-16). Entre 1996 y 1998 se generalizó en Japón el término Continuous Fiber Sheets para designar a los productos usados en la construcción que contengan fibras continuas, tejidos y tendones, preimpregnados con una resina superficial $(17,18)$, tal como se indica en la Tabla 1.

A finales de 1999 el Subcommittee 440F del American Concrete Institute (19) generaliza la clasificación anterior y adopta tres campos principales de aplicación:

a. Rehabilitación: recuperación de la resistencia de la estructura, donde se encuentre comprometida la seguridad local o global debido a la degradación.

b. Refuerzo: refuerzo estructural de elementos para la corrección de anomalías originadas por deficiencias de proyecto o de la capacidad portante por un aumento en la acciones.

c. Sísmico: situaciones de aumento a la resistencia a acciones sísmicas, por medio de la ductilidad y de la resistencia a cortante de los elementos estructurales, permitiendo de este modo la disipación de la energía y un aumento en la capacidad de deformación.

En Europa, a finales de 1996, se formó el “FIB Task Group 9.3: FRP (Fiber Reinforced Polymer) Reinforcement for Concrete Structures". En términos generales, se observa que a nivel internacional en la edificación los sistemas de refuerzo basados en FRP del tipo laminado y tejido flexible, son los más estudiados para el caso de estructuras de hormigón reforzadas exteriormente.

\subsection{Aplicaciones}

Según Meier (14), la primera aplicación de un sistema de refuerzo con FRP sucedió en Europa, en el puente "Kattenbush Bridge" (Alemania) entre 1986 y 1987, donde se utilizaron 20 tiras de un laminado polimérico reforzado con fibras de vidrio (GFRP), y desde 1991 unas 250 estructuras fueron reforzadas en Suiza con laminado polimérico reforzado con fibras de carbono (CFRP), correspondiendo a cerca de $17.000 \mathrm{Kg}$ de material compuesto, sustituyendo a un equivalente de $510.000 \mathrm{Kg}$ de acero. En Alemania y Suiza, la publicación de los primeros documentos de homologación y recomendaciones de proyecto de sistemas laminados de $\operatorname{CFRP}(3,20-22)$, aumentó la confianza a nivel de producción, proyecto y aplicación. En Grecia, la aplicación de los laminados se extendió a la rehabilitación de edificios de carácter histórico $(3,4)$, y en Italia, al refuer- 
zo frente a esfuerzos sísmicos de fábricas y forjados (23). En Japón los sistemas de refuerzo de FRP se desarrollan a finales de los años 80, y son aplicados por primera vez en 1992 en el proceso de refuerzo y confinamiento de elementos en un puente en Tokio (24). La reconstrucción de la ciudad de Kobe, después de haber sido devastada por el sismo de Hanshin en Enero de 1995, es un ejemplo de la importancia de este tipo de materiales en la reparación y refuerzo estructural (18). Priestley (25) se refiere a otras situaciones de revestimiento exterior de pilares de puentes y edificios, para aumentar su capacidad a cortante en casos de refuerzo a sismo. Los primeros ejemplos de rehabilitación de puentes en EE.UU. con mantas de CFRP fueron llevados a cabo en 1994 por Chajes et al. (26). La primera aplicación en España del sistema de refuerzo se llevó a cabo en el puente del Dragó en Barcelona en 1996. Como consecuencia del impacto de un vehículo en la viga de borde, la totalidad de la armadura longitudinal del centro del vano resultó seccionada. Por razones de seguridad estructural y de adecuado comportamiento en servicio, el puente tuvo que ser reforzado con urgencia. Debido a los mayores plazos de ejecución y medios auxiliares requeridos en opciones alternativas estudiadas, se seleccionó la aplicación del refuerzo con materiales compuestos como la más adecuada para la reparación de la estructura.

\section{MODELOS DE CÁlCULO PARA EL DISEÑO A CORTANTE DE VIGAS DE HORMIGÓN CON CFRP}

\subsection{Formulación de Thanasis Triantafillou}

Triantafillou se ha basado en los códigos de diseño en los cuales, para el diseño a cortante de vigas de hormigón armado se supone que la resistencia total está dada por la suma de dos términos: la acción de distintos mecanismos resistentes en el hormigón; y la acción del refuerzo interno. Propone un modelo analítico para obtener la contribución del refuerzo a la resistencia a cortante de la pieza, basado en un factor de eficiencia que depende del mecanismo de fallo. En la Figura 1 se ilustra el mecanismo de transferencia de esfuerzos propuesto, así como la distribución simplificada de tensiones normales a lo largo de una fisura.

En la ecuación [1] se evalúa el cortante soportado por el refuerzo:

\section{[1]}

$\mathrm{V}_{\mathrm{frp}}=\frac{0,9}{\gamma_{\mathrm{frp}}} \cdot \rho_{\mathrm{frp}} \cdot \mathrm{E}_{\mathrm{frp}} \cdot \varepsilon_{\mathrm{frp}} \cdot \mathrm{b}_{\mathrm{w}} \cdot \mathrm{d} \cdot\left(1+\frac{1}{\tan \alpha}\right) \cdot \sin \theta_{\mathrm{frp}}$

donde, $V_{\text {frp }}$ es la resistencia a cortante del

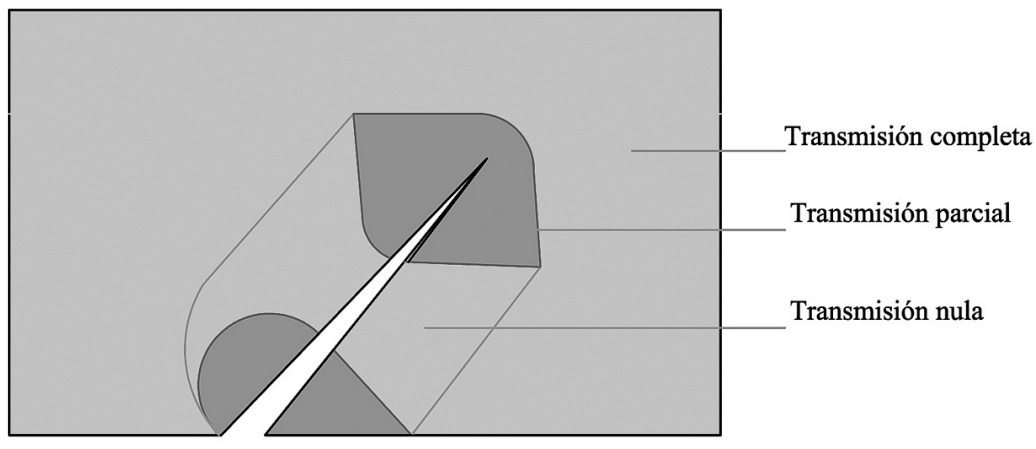

refuerzo de CFRP; $\gamma_{\text {frp }}$ es el coeficiente de seguridad para el refuerzo. Se recomienda tomar 1,15 para CFRP, 1,20 para AFRP y 1,25 GFRP; $\rho_{\text {frp }}$ es la relación del área del refuerzo; $E_{\text {frp }}$ es el módulo de elasticidad del refuerzo de FRP; $\varepsilon_{\text {frp }}$ es la deformación efectiva del refuerzo de FRP; $b w$ es la anchura mínima de la sección a lo largo del canto útil; $\alpha$ es el ángulo formado por los estribos y la dirección longitudinal de la viga; $\theta_{\text {frp }}$ es el ángulo formado por la dirección principal de las fibras y la dirección longitudinal de la viga, medida en sentido horario.

\subsection{Formulación de Malek y Saadatmanesh}

Amir M. Malek y Hamid Saadatmanesh (12) observaron que el ángulo de fisuración se modifica cuando se añade a la viga CFRP. Se determina el efecto que el tejido tiene en la capacidad a cortante de la viga, y en el ángulo de fisuración. Una vez determinado el ángulo de fisuración, se calcula el cortante soportado por la viga mediante las ecuaciones de equilibrio y compatibilidad de deformaciones. La resistencia a cortante de las vigas ordinarias de hormigón armado se calcula basándose en la analogía de la celosía, asumiendo que el hormigón sólo resiste compresiones. El ángulo de inclinación de las fisuras se obtiene mediante la ecuación [2]:

$$
\text { [2] } \quad \theta_{c}=\arctan \sqrt{\frac{\varepsilon_{x}-\varepsilon_{1}}{\varepsilon_{y}-\varepsilon_{2}}}
$$

donde, $x$ es la dirección longitudinal de la viga; $y$ es la dirección perpendicular a $x$; 1 es la dirección principal perpendicular a 2; 2 es la dirección principal paralela a la fisura. Considerando las tensiones que actúan en la viga y usando las ecuaciones de equilibrio, la tensión normal en el hormigón $\left(f_{c}\right)$, la tracción en el armado longitudinal del acero y en el refuerzo de material compuesto $(\Delta N)$ se obtiene como se indica en las ecuaciones [3] y [4].

$$
\begin{gathered}
f_{c}=\frac{V}{b_{v} \cdot h_{v} \cdot \sin \theta_{c} \cdot \cos \theta_{c}} \\
\Delta N=\frac{V}{\tan \theta_{c}}
\end{gathered}
$$


dónde, $V$ es el cortante considerado en la sección; $b_{v}$ es el ancho de la sección encerrada por los estribos; $h_{v}$ es el alto de la sección encerrada por los estribos; $\theta_{c}$ es el ángulo de inclinación de las fisuras.

\subsection{Formulación de Deniaud y Cheng}

Esta formulación evalúa la capacidad a cortante de las vigas usando una combinación del método de las tiras (8) y el método de fricción a cortante (19). El método de la fricción determina que la resistencia a cortante está controlada por el plano más débil de entre todos en los cuales puede ocurrir el deslizamiento. Usando este método, la ecuación general para obtener la resistencia a cortante de una pieza con ángulo de fisuración $\theta_{c}$ es la indicada en la ecuación [5].

$$
V_{n i}=0,25 \cdot k^{2} \cdot b_{w n} \cdot h \cdot \tan \theta_{c}+T_{v} \cdot n_{s}
$$

dónde el valor de $k$ se indica en la ecuación $[6] ; b_{w n}$ y $h$ son el ancho del nervio y el canto total de la viga, respectivamente; $T_{v}$ es la tracción en los estribos; y $n_{s}$ es el número de estribos que cruzan el plano de fallo. El factor $k$ se toma usualmente como 0,5 para hormigón de resistencia normal. Sin embargo, según Loov y Peng (27), el factor $k$ debe ser reducido cuando la resistencia del hormigón aumenta, proponiendo la siguiente relación basándose en ensayos de piezas de hormigón con resistencia entre 20 y 100 MPa.

$$
k=2,1 \cdot\left(\frac{f_{c}^{\prime}}{10^{6} P a}\right)^{-0,4}
$$

Según Tozser y Loov (8), considerar solamente como área de hormigón que participa en la fricción $b_{w n} \cdot h$ para vigas en T es muy conservativo, sugiriendo aproximar el área del ala que interviene en el método con un ángulo de $45^{\circ}$. Para obtener la contribución a cortante del CFRP se rescribe la ecuación [5] incluyendo la contribución del refuerzo de CFRP y la parte del ala de la viga, obteniéndose la ecuación [7].

$$
\text { [7] }
$$

$V_{n}=0,25 \cdot k^{2} \cdot f_{c}^{\prime} \cdot\left(A_{c f} \cdot \tan \theta_{f}+A_{c w} \cdot \tan \theta_{w}\right)+T_{v} \cdot n_{s}+V_{f t p}$

dónde $A_{c f}$ es el área efectiva a cortante del ala; $A_{c w}$ es el área del nervio; y $V_{\text {frp }}$ es la contribución del tejido de fibras. Cuando el tejido es adherido de forma continua, $V_{\text {frp }}$ es el indicado en la ecuación [8]

[8] $V_{f r p}=\frac{d_{f r p} \cdot t \cdot E_{f r p} \cdot \varepsilon_{\max } \cdot R L}{\tan \theta_{w}}$

dónde, $d_{f r p}$ es el canto del nervio de la viga adherido al tejido de fibras; $t$ y $E_{\text {frp }}$ son el espesor y el módulo de elasticidad del refuer- zo, respectivamente; $\theta_{\max }$ es la deformación máxima en el tejido; y $R L$ es la relación entre la longitud que continua adherida en el fallo y la longitud de adhesión inicial. Cuando se aplican bandas de tejido discontinuas para reforzar la viga, la contribución del refuerzo a la resistencia a cortante es la indicada en la ecuación [9].

[9]

$$
\begin{aligned}
& V_{f r p d}=d_{f r p} \cdot t \cdot E_{f r p} \cdot \varepsilon_{\max } \cdot R L \cdot\left(\frac{w_{f r p}}{s_{f r p}}\right)^{2} . \\
& \cdot\left[\frac{\sin \theta_{f r p}}{\tan \theta_{w}}+\left(n_{s}+1\right) \cdot \cos \theta_{f r p}\right] \cdot \sin \theta_{f r p}
\end{aligned}
$$

dónde $W_{\text {frp }}$ y $s_{\text {frp }}$ son el ancho y el espacio entre las bandas de CFRP, medido perpendicularmente a la dirección principal de las fibras; y $\theta_{\text {frp }}$ es el ángulo formado por la dirección principal de las fibras y la dirección longitudinal de la viga. Para obtener la resistencia a cortante de la pieza hay que encontrar el plano más débil de entre todos los posibles planos de fallo. Para esto, los autores desarrollaron un software, encontrando que $\varepsilon_{\max }$ toma un valor aproximado de 0,004 (los resultados de los ensayos fueron entre 0,00468 y 0,00536$), R L$ de 0,85 (entre 0,891 y 0,851$), \theta_{\mathrm{w}}$ de $25^{\circ}$ (entre $25,2^{\circ}$ y $45,4^{\circ}$ ), y $\theta_{f}$ de $15^{\circ}$ (entre $15,3^{\circ}$ y $24,5^{\circ}$ ).

\subsection{Método de Chaallal, Shahawy y Hassan}

En este método se correlaciona la deformación en el refuerzo con la relación de refuerzo a cortante total, consistente en estribos y CFRP. Según este estudio, el refuerzo también produce un incremento en la ductilidad de la pieza, concluyéndose que existe una combinación óptima de estribos y capas de CFRP (28).

Para obtener la contribución del refuerzo a la resistencia a cortante de la viga, se usa el modelo de bielas y tirantes. La contribución del tejido a la capacidad a cortante de la viga se define en la ecuación [10].

$$
V_{f}=f \cdot\left(\frac{A_{f}}{s_{f}}\right) \cdot E_{f}+\varepsilon_{\text {eff }} \cdot d_{f}
$$

donde $A_{f}$ es el área de CFRP igual a $\left(2 \cdot N_{w f}\right)$ donde $N$ es el número de capas de tejido; $t$ es el espesor de cada capa; $w_{f}$ el ancho del material; $\theta_{\text {eff }}$ es la deformación efectiva del refuerzo; $d_{f}$ es la distancia desde la parte superior de la pieza al refuerzo; y $s_{f}$ es el espacio entre las tiras de refuerzo. Tal y como se índica en las ecuaciones [11] y [12].

$$
\begin{gathered}
A_{f}=2 \cdot N \cdot t_{c}+w_{f} \\
t_{f}=t_{c} \cdot N
\end{gathered}
$$


El factor $f$ se obtiene mediante ensayos, basados en que la contribución del refuerzo a la resistencia a cortante es la diferencia entre la resistencia a cortante de la viga reforzada y la resistencia a cortante de la viga original tal como se indica en la ecuación [13].

\section{[13]}

$f=\mid \begin{aligned} & \frac{1+2 \cdot \frac{a}{d}}{12}\left(1000 \cdot \rho_{\text {tot }}-0,6\right) \mathrm{si} \frac{1+2 \cdot \frac{a}{d}}{12}+\left(1000 \cdot \rho_{\text {tot }}-0,6\right) \leq 1 \\ & 1,00 \mathrm{si} \frac{1+2 \cdot \frac{a}{d}}{12}+\left(1000 \cdot \rho_{\text {tot }}-0,6\right)>1,00\end{aligned}$

donde a es la luz a cortante y $d$ el canto útil.

\subsection{Formulación de A. Khalifa y A. Nanni}

Khalifa y Nanni $(9,10,11)$ toman la metodología del $\mathrm{ACl}$ proponiendo que la resistencia nominal a cortante de una sección hormigón armado según la ecuación [14]

$$
V_{n}=V_{c}+V_{s}+V_{f}
$$

donde $V_{c}$ es la resistencia a cortante del hormigón, $V_{s}$ es la resistencia cortante del acero y $V_{f}$ es la contribución al cortante del CFRP. La resistencia nominal a cortante se obtiene multiplicando el valor anterior por un coeficiente $\varphi$, que toma el valor de 0,85 para la contribución del hormigón y las barras de acero y de 0,70 para la contribución al cortante del CFRP [15].

$$
\varphi V_{n}=0,85\left(V_{C}+V_{s}\right)+0,7 V_{f}
$$

La expresión para calcular la contribución del CFRP es similar a la propuesta por el código $\mathrm{ACl}$ para tener en consideración la contribución de las barras de acero [16]:

$$
V_{f}=\frac{A_{f} F_{f e}(\sin \beta+\cos \beta) d_{r}}{s_{r}} \leq\left(\frac{2 \sqrt{f^{\prime}{ }_{c} b_{w} d}}{3}-V_{s}\right)
$$

En la expresión anterior $A_{f}$ es el espesor total de la lámina; $s_{f}$ es la separación entre bandas; $w_{f}$ es el espesor de las láminas. La separación entre bandas está limitada (Figura 2) para evitar una fisuración excesiva, de modo que la separación máxima debe ser menor que la indicada en [17].

$$
S_{f} \leq w_{f}+d / 4
$$

No obstante Khalifa y Nanni observaron que la disposición de bandas en $U$ como refuerzo a cortante mejora la eficiencia del refuerzo cuando la adherencia es un factor crítico de diseño. Por otra parte la resistencia efectiva del CFRP es menor que su resistencia nominal y puede estimarse aplicando un coeficiente $R$ a la resistencia nominal, que depende del modo de rotura.
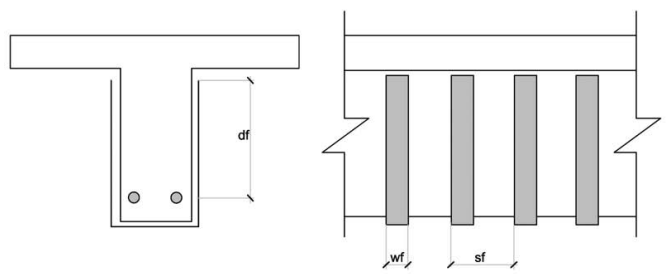

Modo de rotura I (si $r E f \leq 0,70)$ : Se propone un coeficiente de rotura según los resultados experimentales de Triantafillou, modificado, que toma como valor el indicado en la expresión [18].

$\mathrm{R}=0,5622\left(\rho_{f} \mathrm{E}_{f}\right)^{2}-1,2188\left(\rho_{r} E_{f}\right)+0,778$

Modo de rotura II $\left(\right.$ si $\left.r E_{f} \leq 0,70\right)$ : coeficiente de reducción por fallo según el modo 2 . El fallo por despegue depende la longitud efectiva de anclaje determinada en primer lugar:

$W_{f e}=d_{r}-L_{e}$ si las láminas envuelven en forma de $U$ la viga

$W_{f e}=d_{r}-2 L_{e}$ si las láminas están pegadas solamente a los lados de la viga

La expresión final para el coeficiente de reducción $R$, para el modelo de fallo controlado por el despegue del CFRP es el dado por la expresión [19].

\section{ANÁLISISY DISCUSIÓN DE RESULTADOS}

Se analizan los resultados de los modelos que estiman la resistencia a esfuerzo cortante de vigas de hormigón reforzadas con CFRP. Se considera que el cortante es soportado por el hormigón, los estribos de acero, y el tejido de fibras. Para llevar a cabo la comparación de los métodos, se han realizado cálculos sobre un modelo patrón de viga, de $300 \mathrm{~mm} x$ $500 \mathrm{~mm}$ de sección transversal, que recoge la mayor parte de las características de los modelos. Las características geométricas de la viga tipo, así como las cargas aplicadas se muestran en la Figura 3 y en las Tablas 2 y 3.
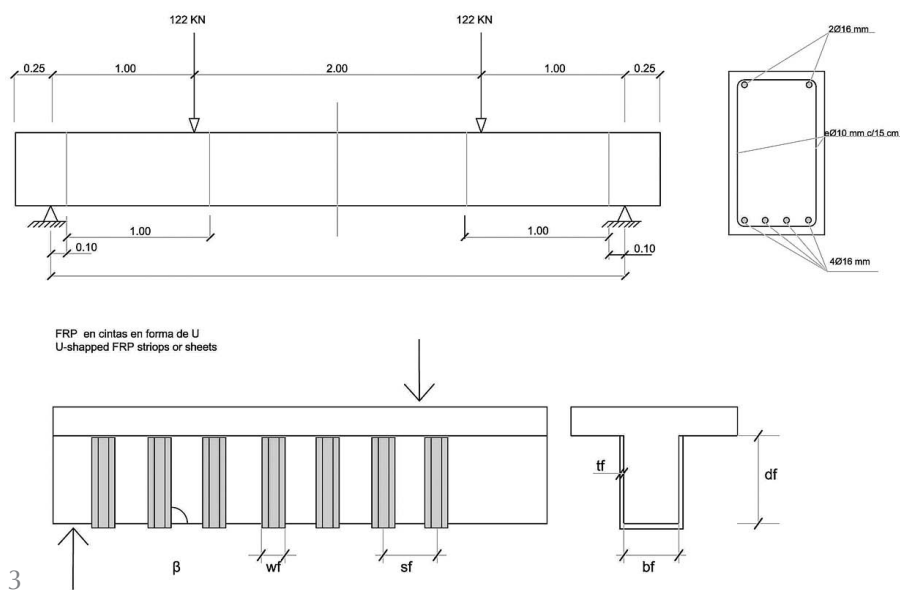
Tabla 2. Geometría de la sección y características de los materiales

\begin{tabular}{ll}
\hline Recubrimiento & $d^{\prime}=3 \mathrm{~cm}$ \\
\hline Altura de la sección encerrada por los estribos & $h_{v}=0,44 \mathrm{~m}$ \\
\hline Ancho de la sección encerrada por los estribos & $b_{v}=0,24 \mathrm{~m}$ \\
\hline Cuantía armadura de tracción & $A_{s}=8,04 \mathrm{~cm}^{2}$ \\
\hline Cuantía armadura de compresión & $A_{s}^{\prime}=4,02 \mathrm{~cm}^{2}$ \\
\hline Área de la sección de los estribos & $A_{v}=1,57 \mathrm{~cm}^{2}$ \\
\hline Separación de la armadura transversal & $s_{v}=0,15 \mathrm{~m}$ \\
\hline$N^{o}$ de estribos que cruzan un plano de fallo & $n_{s s}=1$ \\
\hline Ángulo que forman los estribos & $\alpha=90,00^{\circ}$ \\
\hline Resistencia característica del hormigón a compresión & $f_{c k}=25 \mathrm{MPa}$ \\
\hline Resistencia característica del hormigón a tracción & $f_{c t k}=3,16 \mathrm{MPa}$ \\
\hline Módulo de elasticidad del hormigón & $\varepsilon_{c}=27900 \mathrm{MPa}$ \\
\hline Deformación máxima del hormigón & $e_{c u}=0,0035$ \\
\hline Límite elástico del acero & $f_{y d}=410 \mathrm{MPa}$ \\
\hline Ángulo de fisuración de la sección & $\theta_{c}=38,68^{\circ}$ \\
\hline
\end{tabular}

Tabla 3. Geometría del refuerzo de CFRP

\begin{tabular}{lll}
\hline Espesor del laminado & $t=1,00 \mathrm{~mm}$ \\
\hline $\begin{array}{l}\text { Ángulo que forman la dirección principal de las fibras y } \\
\text { el eje de la viga en sentido horario }\end{array}$ & $\beta=45,00^{\circ}$ \\
\hline $\begin{array}{l}\text { Ángulo que forman la dirección principal de las fibras y } \\
\text { el eje de la viga en sentido anti-horario }\end{array}$ & $\theta=135,00^{\circ}$ \\
\hline $\begin{array}{l}\text { Separación entre las capas de tejido según el eje de la viga } \\
\text { Ancho de la capa de tejido }\end{array}$ & $S_{f}=1,00 \mathrm{~m}$ \\
\hline Módulo de elasticidad longitudinal del laminado & $W_{f}=0,50 \mathrm{~m}$ \\
\hline Módulo de elasticidad transversal del laminado & $E_{f}=155000 \mathrm{MPa}$ \\
\hline Módulo de cizalladura del laminado & $E_{t}=4000 \mathrm{MPa}$ \\
\hline Deformación máxima del laminado & $G_{t t}=6500 \mathrm{MPa}$ \\
\hline $\begin{array}{l}\text { Relación entre la longitud que continua adherida tras el fallo y y } \\
\text { la longitud inicial }\end{array}$ & $\varepsilon_{\max }=0,004$ \\
\hline $\begin{array}{l}\text { Resistencia característica de las fibras en dirección } \\
\text { normal a la fisura }\end{array}$ & $f_{p u}=0,851$ \\
\hline Coeficiente de Poisson de las fibras & $v_{f r p}=0,35$ \\
\hline Coeficiente de seguridad del laminado & $\gamma_{\text {frp }}=1,15$
\end{tabular}

Se ha decidido realizar el estudio con bandas para que la comparación entre métodos sea más homogénea, pues a pesar de la mejora en el comportamiento adherente de las láminas envolventes el comportamiento de sistemas como láminas depende de la orientación de las fibras en la matriz cementante.

\section{1. Ángulo de fisuración de la Pieza}

4. Trayectorias típicas de fisuras de cortante en una viga de hormigón armado.

5. Varición del ángulo de fisuración frente a la cuantía de armadura transversal dispuesta.

(Malek y Saadatmanesh).

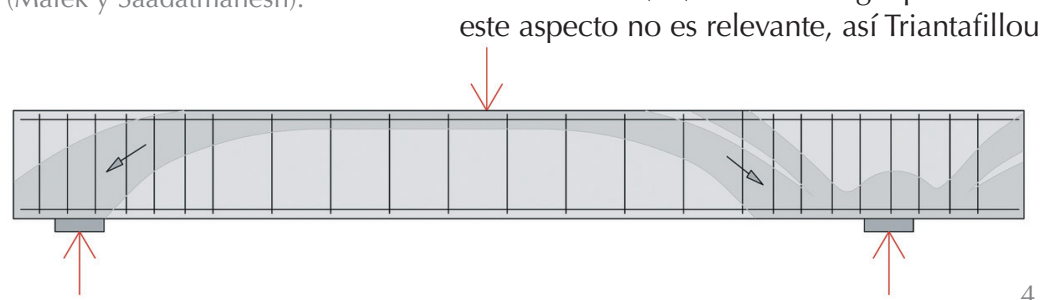

y Khalifa consideran un ángulo de fisuración fijo de $45^{\circ}$. Deniaud y Cheng no indican como calcular el ángulo exacto pero permiten variaciones del mismo entre $25,2^{\circ}$ y $45,4^{\circ}$, que es el intervalo de ángulos de fisuración que obtuvieron en sus ensayos. Chaallal, Shahawy y Hassan no consideran una posible variación en el ángulo de fisuración. Khalifa y Nanni consideran que el ángulo de fisuración se limita mediante la colocación lo suficientemente próxima de las bandas de modo que la fisura siempre es cosida.

En la práctica real, se suele adoptar de forma simplificada un valor de $45^{\circ}$, lo cual se aleja bastante de la realidad física (29). Malek y Saadatmanesh, basándose en las ecuaciones de equilibrio de fuerzas y compatibilidad de deformaciones, presenta un método para estimar el valor de $\theta_{c}$. Según Malek y Saadatmanesh, en una viga de hormigón armado el ángulo de fisuración permanece constante hasta que los estribos alcanzan su límite elástico. Una vez alcanzado, el ángulo de fisuración decrece provocando una fisura mayor. Sin embargo en vigas reforzadas con CFRP, antes y después de que los estribos alcancen su límite elástico, el ángulo de fisuración crece cuando aumenta el espesor del refuerzo (Figura 5), considerando un espesor de refuerzo constante de $1 \mathrm{~mm}$ y una cuantía de armadura transversal dispuesta que varía entre 1,90 y $22,60 \mathrm{~cm}^{2}$.

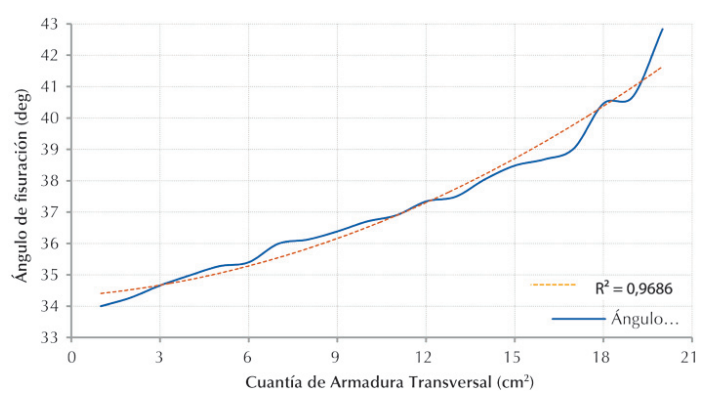

5

Los experimentos realizados por Malek y Saadatmanesh muestran que el cortante soportado por el refuerzo antes de la fisuración de la viga es prácticamente insignificante. Una vez que se produce la fisuración, el hormigón no resiste prácticamente nada de tracción, lo cual produce que tanto el armado interno como el externo tengan que soportar una tracción mayor. La deformación del hormigón crece y debido a que la tensión tangencial es proporcional a la máxima deformación del hormigón, el cortante soportado por el refuerzo, crece.

\section{2. Orientación de las Fibras de CFRP}

Directamente relacionado con el ángulo de fisuración, la orientación con la que se colocan las fibras del refuerzo es un factor a tener en cuenta para el diseño a cortante de refuerzos de CFRP. Considerando un re- 
fuerzo constante de $1 \mathrm{~mm}$ de espesor a cada lado y una cuantía de armadura transversal dispuesta de 10,47 cm²; (estribos de $10 \mathrm{~mm}$ de diámetro separados $15 \mathrm{~cm}$ entre sí), en la Figura 6 se puede observar que si las fibras se colocan sensiblemente paralelas al eje longitudinal de la viga, $\left(\theta_{\text {frp }}\right.$ entre $0^{\circ}$ y $\left.20^{\circ}\right)$, el ángulo que forman las fisuras $\left(\theta_{c}\right)$ se mantiene bajo. Cuando el ángulo de inclinación de las fibras crece también lo hace el de fisuración. Se observa un máximo en la gráfica entorno a $70^{\circ}$. En ese punto las fibras cruzan las fisuras de cortante. En el siguiente tramo (orientación de las fibras entre $70^{\circ}$ y $100^{\circ}$ ) el ángulo de fisuración disminuye (fibras sensiblemente verticales). Si se colocan las fibras con un ángulo comprendido entre $130^{\circ}$ y $160^{\circ}$, el ángulo de fisuración alcanza su valor mínimo (fibras paralelas a las fisuras).

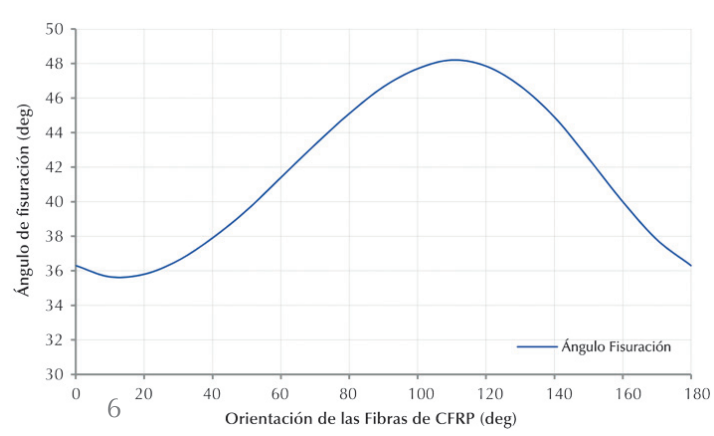

Para todos los métodos de cálculo, los valores máximos de esfuerzo cortante resistido por el refuerzo se alcanza con las fibras dispuestas entre $40^{\circ}$ y $50^{\circ}\left(\theta_{f r p}\right.$ se mide en sentido horario). En ese punto la pieza presenta un ángulo de fisuración $\left(\theta_{c}\right)$ aproximado de $40^{\circ}$, lo que indica que las fibras son sensiblemente perpendiculares a las fisuras (Figura 7). La situación más desfavorable ocurre cuando el ángulo de orientación de las fibras se sitúa en torno a $130^{\circ}$, prácticamente paralelas a las fisuras. En esta situación el refuerzo resiste el mínimo de esfuerzo cortante. La variación del ángulo entre la dirección principal de las fibras y el eje de la viga hace que varíe el cortante resistido por el refuerzo de CFRP según los modelos de cálculo estudiados.

Se observa que, en los resultados obtenidos, con la formulación de Triantafillou así como con la de Khalifa y Nanni los valores de inclinación de las fibras más allá de los $90^{\circ}$ proporcionan valores de resistencia a cortante muy bajos, probablemente el método no está diseñado para calcular inclinaciones que no son coherentes con la lógica del cálculo estructural. Malek y Saadatmanesh, tienen valores, en torno al $60 \%$, inferiores al obtenido aplicando el método de Deniaud y Cheng. Mención aparte merecen Chaallal, Shahawy y Hassan que no tienen en cuenta el factor de la orientación de las fibras para el cálculo.

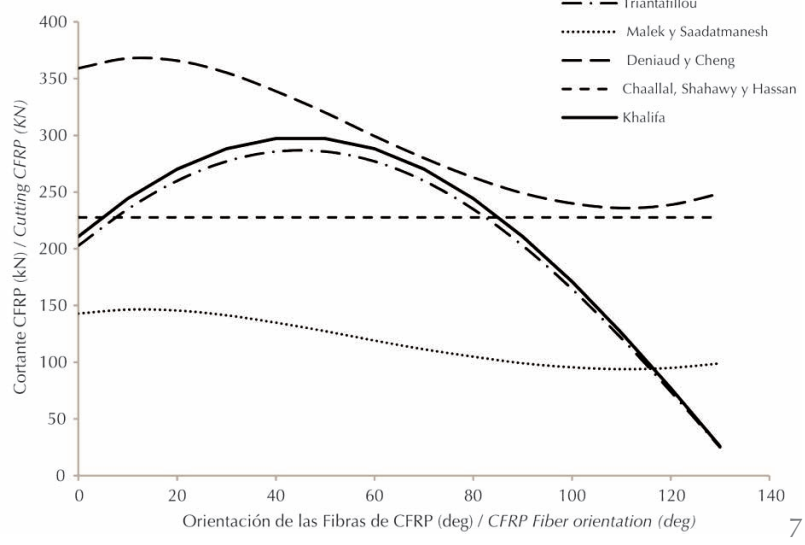

\subsection{Deformación en el tejido de CFRP}

Algunos investigadores consideran que la contribución a la resistencia a cortante del tejido de FRP depende de la deformación efectiva del tejido. Dicha deformación depende de las condiciones de adherencia entre el hormigón y el refuerzo, de la rigidez del refuerzo (área multiplicada por el módulo elástico) y del armado interno de la pieza. Es de esperar, en refuerzos llevados a cabo con materiales de mayor rigidez, una deformación efectiva menor y un modo de fallo controlado por el despegue del refuerzo y no por la rotura a tracción de las fibras (30). Por otra parte, en los materiales de módulo elástico más bajo, como es la fibra de vidrio, se necesitan grandes deformaciones para que se aproveche su resistencia total (Tabla 4).

Tabla 4. Valores típicos de las propiedades de las fibras

\begin{tabular}{lccc}
\hline \multicolumn{1}{c}{ Tipo de fibra } & $\begin{array}{c}\text { Módulo de elasticidad } \\
\text { a tracción GPa }\end{array}$ & $\begin{array}{c}\text { Resistencia a } \\
\text { tracción MPa }\end{array}$ & $\begin{array}{c}\text { Deformación axial } \\
\text { máxima en rotura \% }\end{array}$ \\
\hline $\begin{array}{l}\text { Carbono tipo 1 } \\
\text { (módulo bajo) }\end{array}$ & 170 & 1380 & 0,90 \\
\hline $\begin{array}{l}\text { Carbono tipo 2 } \\
\text { (módulo alto) }\end{array}$ & 380 & 1720 & 0,40 \\
\hline $\begin{array}{l}\text { Carbono tipo 3 } \\
\text { (módulo muy alto) }\end{array}$ & 760 & 2210 & 0,30 \\
\hline Vidrio (E-glass) & 81 & 3450 & 4,88 \\
\hline Vidrio (S-glass) & 89 & 4590 & 5,70 \\
\hline $\begin{array}{l}\text { Aramida } \\
\text { (alta tenacidad) }\end{array}$ & 83 & 3620 & 2,00 \\
\hline $\begin{array}{l}\text { Aramida } \\
\text { (módulo alto) }\end{array}$ & 131 & $3620-4140$ & 2,00 \\
\hline $\begin{array}{l}\text { Aramida } \\
\text { (módulo muy alto) }\end{array}$ & 186 & 3450 & \\
\hline
\end{tabular}

Para obtener la deformación del tejido los autores estudiados se postulan de dos formas distintas. Por una parte, Chaallal y Triantafillou, proponen expresiones basadas en los datos experimentales disponibles, obteniendo a partir de ellos ecuaciones ajustadas estadísticamente. Por otra parte, Triantafillou recomienda limitar el valor de $\rho_{\text {frp }} \cdot E_{\text {frp }}$ a 0,40 GPa para determinar el área límite a partir del cual un incremento en el área de refuerzo deja de ser positiva. Khalifa y Nanni considera que el valor de $\rho_{\text {frp }} \cdot E_{\text {frp }}$ condiciona el modo de fallo. Así, consideran que valores inferiores a $0,7 \mathrm{GPa}$ pueden
6. Relación entre la orientación de las fibras y el ángulo de fisuración de la pieza.

(Malek y Saadatmanesh).

7. Relación entre la orientación de las fibras y el esfuerzo cortante resistido por el refuerzo de CFRP. 


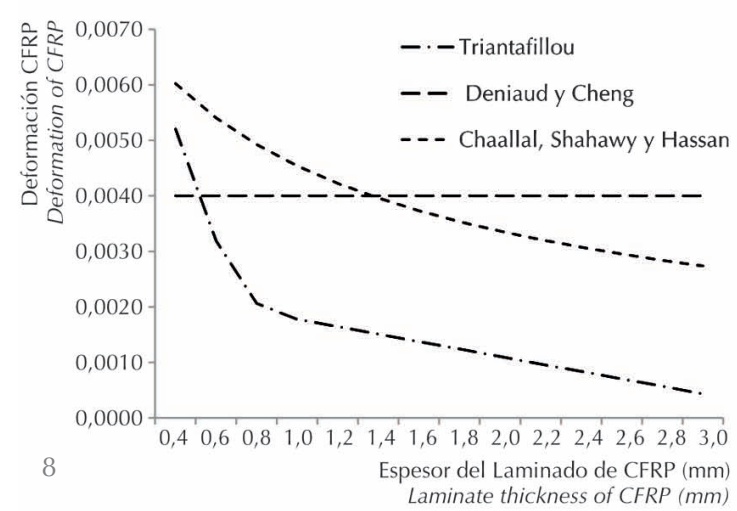

8. Cálculo de la deformación del CFRP para cada espesor de refuerzo dispuesto y cuantía de armadura transversal.

9. Relación entre el Cortante soportado por el tejido de CFRP y el espesor de tejido dispuesto. fallo por adherencia. dan lugar a un fallo por rotura de la lámina y valores superiores pueden dar lugar a un

En la viga tipo que se está analizando el valor de $\rho_{\text {frr }} \cdot E_{\text {frp }}$ es 1,03 GPa. Si se considera un valor del área del refuerzo tal que $\rho_{\text {frp }} \cdot E_{\text {frp }}$ sea igual a 0,4 GPa se obtiene una contribución del refuerzo a la resistencia a cortante, sólo un 4 por ciento inferior a la obtenida con $\rho_{\text {frp }} \cdot E_{\text {frp }}$ igual a 1,03 GPa. La limitación supone una reducción significativa del cortante soportado por el refuerzo. Como se observa en la Figura 10 una vez alcanzado un área máxima de refuerzo, aunque aumente el espesor del refuerzo, el cortante soportado por el tejido no aumenta, sino que disminuye.

Por otra parte, Deniaud considera que el cortante soportado por las fibras depende de la deformación del tejido y no proponen expresiones para obtener dicha deformación. Considera una deformación admisible máxima en el tejido, obtenida experimentalmente, del 4 por mil $(0,004)$. El Comité 440 del $\mathrm{ACl}$ recomienda como deformación límite en las fibras de carbono 0,004, mientras que el "Japan Building Disaster Prevention Association" recomienda 0,007.

\subsection{Espesor del Tejido de CFRP}

Los métodos estudiados muestran que el cortante soportado por el tejido aumenta cuando crece su espesor (Figura 8 y 9). En el método de Triantafillou se llega a un valor máximo asociado a la limitación de deformación del tejido indicada por dicho investigador. El método de Challal, Shahawy y Hassan también considera dos limitaciones en la cuantía de refuerzo dispuesto. Con ellas el autor intenta evitar un fallo prematuro a compresión del hormigón. En primer lugar se limita la mejora del refuerzo al 33\% del cortante soportado por la viga original y en segundo se limita la relación entre la rigidez del acero y la del refuerzo de CFRP a 2. En la gráfica de la Figura 10 se observa un punto de inflexión $(2,4 \mathrm{~mm}$ de espesor de

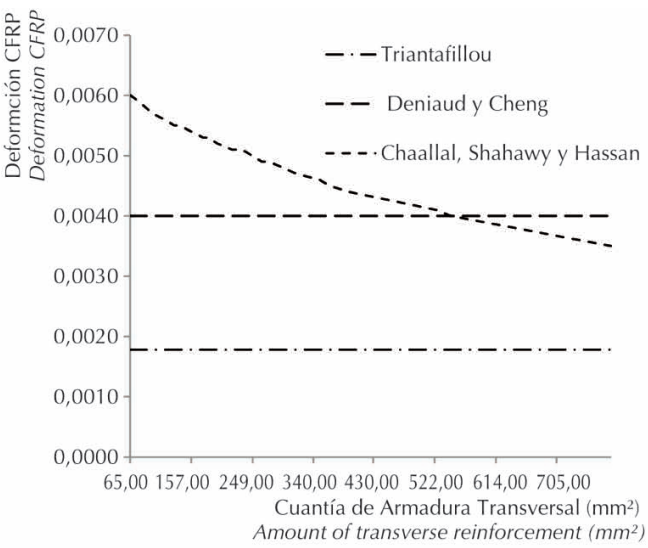

refuerzo) a partir del cual se tienen en cuenta estas limitaciones. El método de Deniaud y Cheng resulta ser el que tiende a obtener una menor aportación a la resistencia del CFRP, mientras el de Malek y Saadatmanesh al igual que Khalifa son los que tienden a incrementar de manera más significativamente la influencia del espesor del laminado.

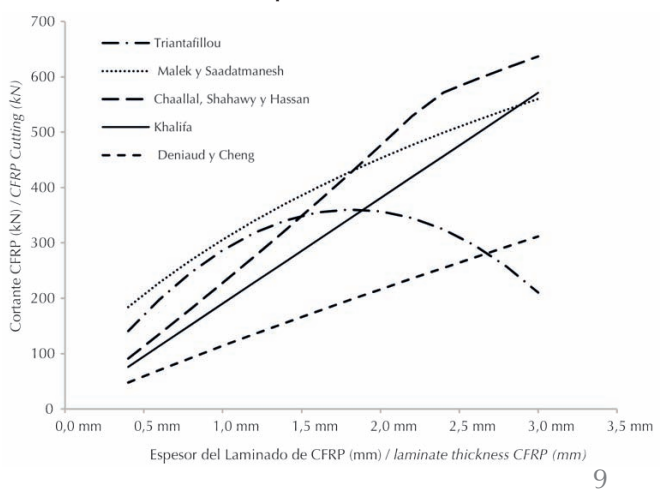

\subsection{Armadura Transversal}

En la Figura 10 realizada considerando un refuerzo constante de $1 \mathrm{~mm}$ de espesor a cada lado y una cuantía de armadura transversal dispuesta desde 1,90 $\mathrm{cm}^{2}$ (estribos de $6 \mathrm{~mm}$ de diámetro separados $30 \mathrm{~cm}$ ) a $22,60 \mathrm{~cm}^{2}$ (estribos de $12 \mathrm{~mm}$ de diámetro separados 10 $\mathrm{cm})$, se muestra la relación entre la resistencia a cortante del refuerzo y la armadura transversal dispuesta. Se observa que el cortante soportado por el refuerzo de CFRP disminuye al aumentar el armado interno de la viga. Malek y Saadatmanesh basan su método en la compatibilidad de deformaciones de la viga original con las deformaciones que presentará la viga reforzada, por tanto, una viga fuertemente armada se deformará menos, luego necesitará menos colaboración del refuerzo ante un aumento de carga y por consiguiente de esfuerzo cortante y deformación.

Deniaud y Cheng basan su método en los ensayos realizados. Tienen en cuenta la armadura transversal indirectamente al considerar el ángulo de fisuración de la pieza para calcular la contribución del refuerzo. Un aumento de la armadura transversal trae aparejado un au- 
mento del ángulo de fisuración. Fisuras más verticales implican menos tensión tangencial, es decir, se moviliza menos la capacidad del refuerzo. Según el estudio de Chaallal, Shahawy y Hassan la contribución del material compuesto a la resistencia a cortante depende de los estribos de la viga original. Existe una combinación óptima de espesor de refuerzo y armadura transversal. Sin embargo parecen obviar la importancia de colocar las fibras en la dirección correcta y solo consideran la resistencia del refuerzo en el caso en el que estén orientadas verticalmente.

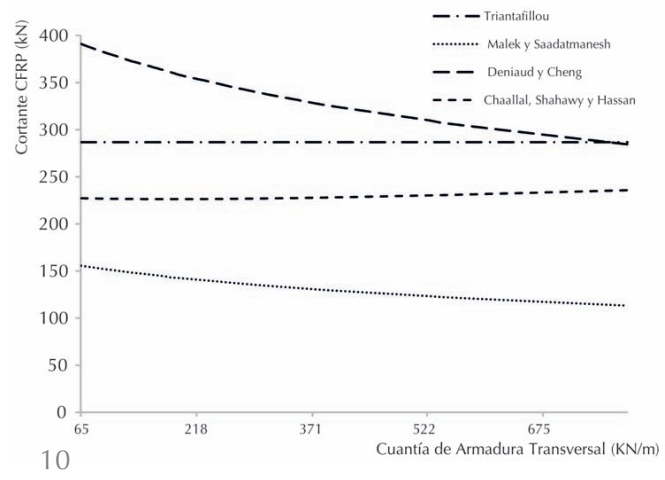

En definitiva los parámetros considerados en el cálculo de la contribución a la resistencia del esfuerzo cortante de CFRP difieren considerablemente entre los autores estudiados (Tabla 5). portamiento de la viga por el refuerzo, y calcular previamente el ángulo de fisuración. De las investigaciones consultadas, solo Khalifa y Nanni cuantifican el primer aspecto y Malek y Saadatmanesh el segundo.

3) La dirección principal de las fibras debe ser sensiblemente perpendicular a las fisuras de cortante. Si esta con Figuración no es posible se recomienda una disposición vertical de las fibras.

4) El ángulo de inclinación de las fisuras y el cortante resistido por el refuerzo, dependen del ángulo de inclinación de las fibras. Cuando las fibras se orientan formando un ángulo de $130^{\circ}$, el cortante soportado por el refuerzo es máximo debido a que en este caso las fisuras son prácticamente perpendiculares a las fibras.

5) Todos los investigadores estudian cómo influye la deformación del refuerzo en sus modelos de cálculo, por tanto es un factor a tener en cuenta para el diseño de refuerzos. Los resultados obtenidos aplicando el modelo de Triantafillou y Chaallal tienden a obtener menores resistencias que el resto, Malek y Saadatmanesh se basan en la compatibilidad de desplazamientos para elaborar su método, y Deniaud y Cheng toman un valor constante, con un buen ajuste a los valores experimentales obtenidos.
10. Cálculo del incremento de esfuerzo cortante resistido por la viga tipo.

Tabla 5. Comparación entre los modelos de cálculo estudiados

\begin{tabular}{|c|c|c|c|c|c|}
\hline & $\begin{array}{c}\text { Formulación de } \\
\text { Thanasis C. Triantafillou }\end{array}$ & $\begin{array}{c}\text { Formulación de } \\
\text { Malek y Saadatmanesh }\end{array}$ & $\begin{array}{l}\text { Formulación de } \\
\text { Deiaud y Cheng }\end{array}$ & $\begin{array}{c}\text { Formulación de } \\
\text { Chaallal, Shanawy y Hassan }\end{array}$ & $\begin{array}{l}\text { Formulación de } \\
\text { Khalifa y Nanni }\end{array}$ \\
\hline Ángulo de fisuración & $\sqrt{(1)}$ & $\sqrt{ }$ & - & - & - \\
\hline Orientación de las fibras de CFRP & $\sqrt{ }$ & $\sqrt{ }$ & - & - & $\sqrt{ }$ \\
\hline Deformación del tejido de CFRP (2) & - & - & $\sqrt{ }$ & - & - \\
\hline Limitación de la cuantía de CFRP ${ }^{(2)}$ & $\sqrt{ }$ & - & - & - & - \\
\hline Espesor del refuerzo de CFRP ${ }^{(2)}$ & $\sqrt{ }$ & - & $\sqrt{ }$ & $\sqrt{ }$ & $\sqrt{ }$ \\
\hline Armadura transversal & - & - & - & $\sqrt{ }$ & $\sqrt{ }$ \\
\hline
\end{tabular}

(1) Incluye el ángulo de fisuración mediante datos experimentales. Además Thanasis y Triantafillou han propuesto cambier el factor

$(1+\operatorname{cotag} \alpha)$ que figura en este artículo por $(\operatorname{cotag} \theta+\operatorname{cotag} \alpha)$ para recoger este efecto.

(2) La deformación del tejido, la limitación en la cuantía del refuerzo y un límite en su espesor máximo están íntimamente ligados, al recoger implícitamente múltiples efectos como el despegue de láminas.

\section{CONCLUSIONES}

Con el desarrollo de este trabajo de investigación del estudio del refuerzo a esfuerzo cortante de vigas de hormigón armado mediante refuerzo de CFRP se pueden deducir las siguientes conclusiones:

1) El refuerzo a cortante de vigas de hormigón armado con CFRP es un fenómeno complejo, afectado por múltiples variables que recogen sólo parcialmente los distintos modelos, no obstante el comportamiento a cortante mejora en todos los casos observados gracias a la adhesión de polímeros reforzados con fibras de carbono (CFRP).

2) En el diseño del refuerzo se tendrá que tener en cuenta la modificación del com-
6) La contribución del refuerzo a la resistencia a cortante de la viga depende de los estribos de acero existentes en la viga original. Así, el refuerzo es menos efectivo cuando la viga está fuertemente armada a cortante.

7) La resistencia a cortante de la viga reforzada se incrementa cuando lo hace el espesor del refuerzo. Esta relación es casi lineal cuando la viga no está fisurada. Según Triantafillou dado el refuerzo interno de la viga, existe un espesor óptimo para el cual el incremento que se produce en la resistencia a cortante es máximo. Se necesitan ensayos, para cada tipo de sección, para obtener la relación existente entre el incremento en la resistencia a cortante, el refuerzo interno, y el refuerzo externo, obteniéndose a partir de ésta el espesor óptimo. 


\section{BIBLIOGRAFÍA}

(1) Escudero, D.: "Materiales Compuestos. Aplicaciones". Informes de la Construcción, vol. 52, n. 472 (2001), pp. 45-52.

(2) Recuero, A.; Gutiérrez, J.; Miravete, A. y Cuartero, J.: "Refuerzo de estructuras con composites avanzados". Informes de la Construcción, vol. 49, n. ${ }^{\circ} 452$ (1997), pp. 39-50.

(3) Triantafillou, T. C. \& Fardis, M. N.: "Advanced composites for strengthening of historic structures", proceedings of IABSE Symposium on structural preservation of the architectural heritage, Rome, Italia: 541-548, 1993.

(4) Triantafillou, T. C.: "Innovative strengthening of masonry monuments with composites", proceedings of the 2 nd International Conference on Advanced Composite Materials in Bridges and Structures (ACMBS-II), Montreal, Canada, Agosto: 473-480, 1996.

(5) Triantafillou, T. C.: "Shear Strengthening of Reinforced Concrete Beams Using Epoxi-Bonded FRP Composites", ACl Structural Journal, vol. 95 (2) (1998), pp. 107-115.

(6) Triantafillou, T. C.; Deskovic, N: "Innovative prestressing with FRP sheets: Mechanics of short-term behaviour", Journal of Engineering Mechanical, vol. 117 (7) (1991), pp. 1652-1672.

(7) Triantafillou, T. C.; Deskovic, N.; Deuring, N.: "Strengthening of concrete structures with prestressed fiber reinforced plastic sheets". ACI Structural Journal, vol. 89 (3) (1992), pp. 235-244.

(8) Deniaud, C.; Cheng, J. J. R.: "Shear Behavior of Reinforced Concrete T-Beams with Externally Bonded Fiber-Reinforced Polymer Sheets". ACI Structural Journal, vol. 98 (3) (2001), pp. 386-394.

(9) Khalifa, A.; Gold, W. J.; Nanni, A.; Aziz, A.: "Contribution of Externally Bonded FRP to Shear Capacity of RC Flexural Members with FRP". Journal of Composites for Construction, ASCE, vol. 2, n. ${ }^{\circ} 4$ (1998), pp. 195-203.

(10) Khalifa, A.; Nanni, A.: "Improving shear capacity of existing RC T-section beams using CFRP composites". Cement and Concrete Composites, 22 (2000), pp. 165-174.

(11) Khalifa, A.; Nanni, A.: "Rehabilitation of rectangular simply supported RC beams with shear deficiencies using CFRP composites". Construction and Building Materials, 16 (2002), pp. 135-146.

(12) Malek, A. M.; Saadatmanesh, H.: "Ultimate Shear Capacity of Reinforced Concrete Beams Strengthened with Web-Bonded Fiber-Reinforced Plastic Plates". ACI Structural Journal, vol. 95, n. ${ }^{\circ}$ (1998), pp. 391-399.

(13) Malek, A. M.: Analytical study of reinforced concrete beams strengthened with fiber reinforced plástic plates (fabrics), Ph. D. Thesis, University of Arizona, Tucson, Arizona, USA: 163. 1997.

(14) Oehlers D. J.; Moran J. P.: "Premature Failure of Externally Plated Reinforced Concrete Beams". Journal of Structural Engineering, ASCE, vol. 116, n. ${ }^{4}$, (1990), pp. 978-995.

(15) Saadatmanesh, H.; Ehsani M. R.: "RC Beams Strengthened with GFRP Plates. I: Experimental Study". Journal of Structural Engineering, ASCE, vol. 117, n. ${ }^{\circ} 11$ (1991), pp. 3417-3433.

(16) Arduini, M.; D’Ambrisi, A; Ditommaso, A.: "Shear failure of concrete beams reinforced with FRP plates". Infrastructure: New Materials and Methods of Repair, ASCE, New York, (1994), pp. 123-130.

(17) JCl, TC952: Continuous fiber reinforced concrete. Japan Concrete Institute, Tokyo, Japan, 1998.

(18) JSCE: "Recommendation for design and construction of concrete structures using continuous fiber reinforcing material". JSCE, Concrete Engineering Series, n. ${ }^{\circ} 23$, Tokyo, Japan: 325. 1997.

(19) $\mathrm{ACI}$ 440F: Guidelines for the selection, design and installation of fiber reinforced polymer (FRP) systems for externally strengthening concrete structures. American Concrete Institute, ACI Committee 440, Sub Committee 440F, febrero, 1999.

(20) D0128: Nachträgliche verstärkung von bauwerken mit CFK-Lamellen. Structure Reinforcement Using CFRP, SIA, Zurich, Septiembre, 1995.

(21) Rostasy, F. S.: Assement of suitability of CRP plates from the S\&P CRP system for use as adhesivebonded reinforcement to strengthen concrete constructional elements and bases of assessment for their general approval by the construction supervisory authorities. Expert opinion n. ${ }^{\circ}$ 98/0322, S\&P Reinforcement, TU Braunschweig, 1998.

(22) Rostasy, F. S.: "Beurteilung der eignug von CFK amellen des systems Sika Carbodur ais Klebewehrung für die verstärkung von betonbauteilen sowie bemessungsgrunndlagen für die allgemeine bauaufsichtliche zulassung". Gutachen Nr. 97/0250, 14.01.1997, Sika, TU Braunscweig, 1997.

(23) Spena, F. R. et al.: L'uso di materiali compositeper il cosolidamento delle structure. Centro Internazionale di Studi di Archtettura Andrea Palladio, Vicenza, Italia, 1995.

(24) Meier, U.: Repair using advanced composites, proceedings of the International Conference of Composite Construction - Conventional and Innovative, IABSE, Innsbruck, Austria, Septiembre: 113-124, 1997.

(25) Priestley, M. J. N.; Seible, F.; Fyfe, E.: Column seismic retrofit using fibreglass/epoxy jackets, Proceedings of the 1st International Conference on Advanced Composite Materials in Bridges and Structures, ACMBS-I, Sherbrooke, Canada: 287-298, 1992.

(26) Chajes, M.; Thomson, T.; Januszka, T.; Finch, W. W.: "Flexural strengthening of concrete beams using externally bonded composite materials". Construction And Building Materials, vol. 8, n. 3 (1994), pp. 191-201.

(27) Loov, R.; Peng, L.: The influence of concrete strength on shear-friction based design of reinforced concrete beams. In: International Conference on HPHSC, Perth Australia, Canada. Proceedings: 505-519, (1998).

(28) Chaallal, O.; Shahawy, M.; Hassan, M.: "Performance of Reinforced Concrete T-Girders Strengthened in Shear with Carbon Fiber-Reinforced Polymer Fabric". ACI Structural Journal, vol. 99, n. ${ }^{\circ}$, (2002), pp. 335-343.

(29) Perera-Velamazan, R.; Vique, J.; Arteaga-Iriarte, A., de Diego-Villalón, A.: "Diseño a Cortante de vigas de hormigón armado con armadura de acero y armadura externa de FRP mediante el mecanismo de bielas y tirantes y algoritmos genéticos". Hormigón y Acero, vol. 60, n. ${ }^{\circ} 254$ (2009), pp. 65-83.

(30) Olivares, S.; Galán, C.; Roa, J.: "Los composites: características y aplicaciones en la edificación". Informes de la Construcción, vol. 54, n. ${ }^{\circ} 484$ (2003). 